\section{Polypseudophakia for cataract surgery: 10-year follow-up on safety and stability of two poly-methyl- methacrylate (PMMA) intraocular lenses within the capsular bag}

${ }^{1}$ Sussex Eye Hospital, Brighton, UK

${ }^{2}$ Ophthalmology

Department, Cairo

University, Cairo, Egypt

${ }^{3}$ Brighton and Sussex Medical School, Brighton, UK

${ }^{4}$ Tongdean Eye Clinic, Hove, UK

Correspondence: CSC Liu, Ophthalmology

Department, Sussex Eye Hospital, Eastern Road, Brighton BN2 5BF, UK Tel: + 44 (0) 1273606126 ; Fax: + 44 (0) 1273693674

E-mail: cscliu@aol.com

Received: 17 November 2010

Accepted in revised form: 20 March 2011

Published online: 13 May 2011

\begin{abstract}
Purpose To report the long-term follow-up (more than 10 years) of three patients who have undergone polypseudophakia phacoemulsification cataract surgery. Methods A case series of three patients and four procedures. Two poly-methylmethacrylate (PMMA) intraocular lenses (IOLs) were placed within the capsular bag (P359UV, Storz, Tuttlingen, Germany). No complications occurred peri-operatively. A full ophthalmological examination was performed at 10-year follow-up, looking for decentration, tilt, and complications of interlenticular opacification (ILO) between the IOLs.

Results Inferior ILO with Elschnig pearls was observed in only one case, and was likely to be visually insignificant. No ILO was observed in the other three eyes. In one patient, the piggyback IOL had been displaced 1-2 mm nasally, but there was no tilt of the IOLs, with the haptics remaining well aligned. There was no displacement or tilt of the piggyback IOL in the other three cases. Corneal endothelial cell count (SP-2000P, Topcon, Tokyo, Japan) was above $1000 \mathrm{cell} / \mathrm{mm}^{2}$ in all cases.

Conclusions With the introduction of foldable IOLs, the piggyback IOL is usually placed in the sulcus, but we have shown good long-term stability and minimal complications of dual PMMA IOLs placed within the bag. Eye (2011) 25, 1090-1093; doi:10.1038/eye.2011.107; published online 13 May 2011
\end{abstract}

A Gomaa ${ }^{1,2}, \mathrm{RMH}$ Lee $^{1}$ and CSC Liu',3,4
Keywords: polypseudophakia; piggyback; intraocular lens; cataract; phacoemulsification; PMMA

\section{Introduction}

Polypseudophakia can be defined as the implantation of two or more intraocular lenses (IOL) following phacoemulsification cataract extraction. This can be primary when the IOLs are placed together during surgery, or secondary when additional IOLs are placed at a later date. Polypseudophakia was first described by Gayton in 1993. ${ }^{1}$

Polypseudophakia can be complicated by interlenticular opacification (ILO), commonly developing within 6 months to 2 years. ${ }^{2}$ We previously published the results of our cohort of patients who underwent primary polypseudophakia, using dual in-the-bag polymethyl-methacrylate (PMMA) IOLs, where we observed a new form of ILO (Case 1) after 3 years, and recommended longer-term follow-up of these patients. ${ }^{2}$ We now present the 10-year postoperative findings of our patients, and assess the stability of polypseudophakia cataract surgery. To our knowledge, there has been no previous published study of the long-term outcome of patients who underwent polypseudophakia for cataract surgery at 10 years.

Case reports

Of fifteen patients who underwent polypseudophakia surgery, three were available 
for review. There were no complications during surgery, and all patients received the same model of PMMA IOL (P359UV, Storz, Tuttlingen, Germany). One patient underwent primary polypseudophakia in both eyes, and two patients had secondary polypseudophakia in one eye. Clinical findings are summarized in Table 1.

\section{Case 1}

A 57-year-old woman with a history of Laurence-Moon syndrome, nystagmus, retinitis pigmentosa, and hypermetropia in both eyes underwent cataract extraction for both eyes with primary polypseudophakia in 1999. Two IOLs were implanted in her right eye (both $+16.00 \mathrm{D})$ and left eye $(+16.00,+15.50 \mathrm{D})$. Her vision did not improve following surgery in either eye, despite technically successful procedures. At her 2-week and 15-month post-operative follow-up, a type of ILO was observed in both eyes in the form of centrally located pigment deposits surrounded by Newton's rings. ${ }^{2}$ At 10-year follow-up, posterior capsular opacification was observed in the right eye, and the previously observed central ILO was no longer observed in either eye although in the left eye inferior ILO was noted, but it remained off axis (Figure 1).

\section{Case 2}

An 87-year-old woman with central corneal shagreen dystrophy had phacoemulsification cataract extraction with IOL implantation (+ 17.5 D) in her right eye in 1998, but developed a post-operative refractive surprise (Table 1). A piggyback IOL ( $+4.0 \mathrm{D})$ was inserted into the capsular bag in 1999, with a good post-operative outcome subjectively. At 10-year follow-up, the piggyback lens was nasally displaced by $1-2 \mathrm{~mm}$ (Figure 2).

\section{Case 3}

A 48-year-old woman had phacoemulsification cataract extraction with IOL implantation $(+20.0 \mathrm{D})$ in her right eye in 1995 (pre-operative refraction $+6.75 /-1.25 \times$ $456 / 12$ ), but remained hypermetropic (Table 1). A piggyback IOL $(+5.00 \mathrm{D})$ was inserted into the capsular bag in 1999, with a good post-operative outcome subjectively. At 10-year follow-up, no decentration or tilt of the IOL, and no ILO was observed.

\section{Discussion}

Polypseudophakia can be used during phacoemulsification cataract surgery in patients with high levels of refraction, and can also be used for






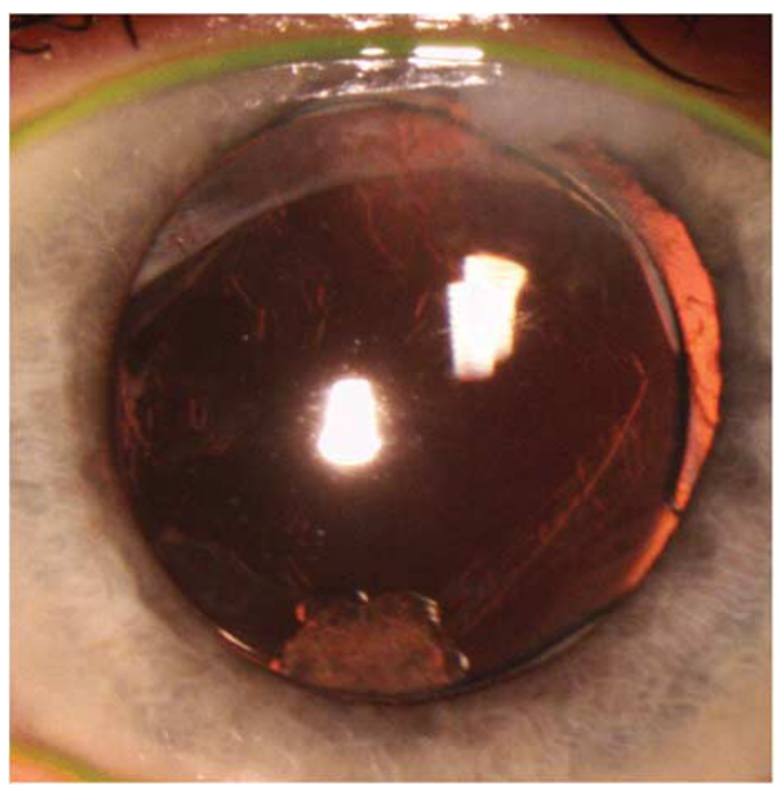

Figure 1 Slit lamp photograph of the left eye of Case 1 showing inferior Elschnig pearls, ILO after pupil dilatation. These are unlikely to be visually significant.

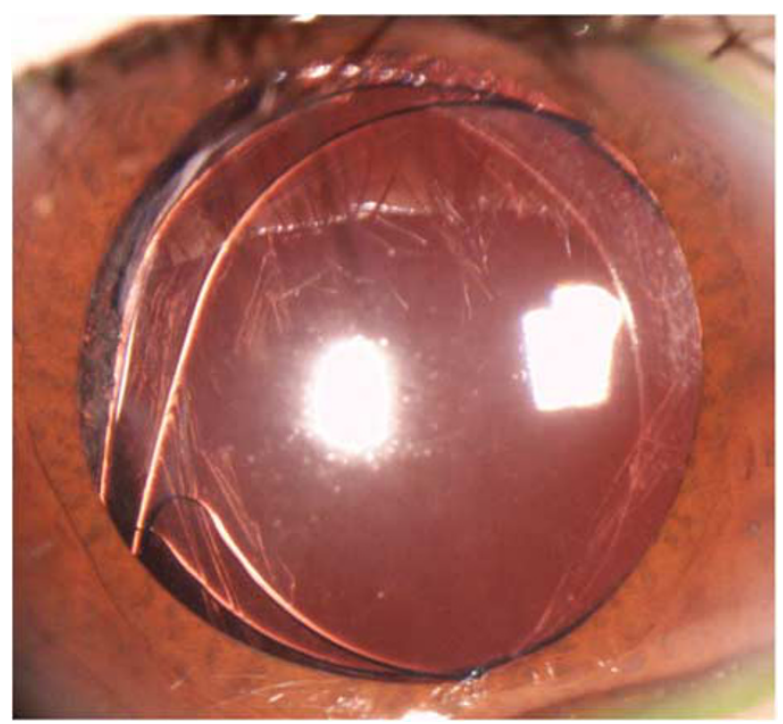

Figure 2 Slit lamp photograph of Case 2 demonstrating nasal displacement by $1-2 \mathrm{~mm}$.

correction of refractive surprises, or to add multifocality, ${ }^{3}$ or a toric correction. ${ }^{4}$ In Case 1, primary

polypseudophakia was chosen instead of a single highpowered IOL, as they were less readily available in 1999, and would often have to be custom made. We have also found from theoretical calculations that polypseudophakia offers better optical quality due to a reduction in spherical aberration ${ }^{5}$ than a single highpowered IOL.
We observed no tilt of the IOLs with the haptics remaining well aligned in all cases. Of our case series, only one patient showed decentration of the IOL, but her visual acuity was good, despite the presence of central corneal dystrophy. We also observed that the patients' central corneal endothelial cell density (SP-2000P, Topcon, Tokyo, Japan) were reasonable, which provides much needed long-term evidence to support the use of polypseudophakia, following corneal graft surgery. ${ }^{6,7}$

ILO is a well-recognized complication of polypseudophakia ${ }^{8,9}$ associated with a reduction of visual acuity and hyperopic shift. We could not confirm this in our patient with ILO, but it was unlikely to be visually significant being off axis. In our previous study, we observed a new form of ILO, but this had since resolved and was not present in our other two patients. However, with a trend in using foldable IOLs to minimize wound size, placing one IOL in the sulcus has been found to minimize ILO.

In summary, we have demonstrated long-term stability of polypseudophakia following phacoemulsification cataract surgery using dual PMMA IOLs in the bag, with the haptics parallel one set to another. Corneal endothelial cell density was good, although our follow-up cohort was small. The IOLs remained well centered with minimal decentration, and complications of ILO were likely to be visually insignificant.

\section{Summary}

\section{What was known before}

- Polypseudophakia cataract surgery was initially described in a case of extreme hypermetropia, but its role has extended to include the use of multifocal and toric IOLs, or following post-corneal graft astigmatism.

- ILO is a commonly observed complication that can result in long-term reduction of BCVA and hyperopic shift.

What this study adds

- This is the first long-term follow-up case series of patients who have undergone polypseudophakia cataract surgery.

- We report good long-term stability of polypseudophakia, and ILO described was thought to be visually insignificant.

\section{Conflict of interest}

The authors declare no conflict of interest.

\section{References}

1 Gayton JL, Sanders VN. Implanting two posterior chamber intraocular lenses in a case of microphthalmos. J Cataract Refract Surg 1993; 19: 776-777.

2 Eleftheriadis H, Sciscio A, Ismail A, Hull CC, Liu C. Primary polypseudophakia for cataract surgery in hypermetropic 
eyes: refractive results and long term stability of the implants within the capsular bag. Br J Ophthalmol 2001; 85(10): 1198-1202.

3 Akaishi L, Tzelikis PF, Gondim J, Vaz R. Primary piggyback implantation using the Tecnis ZM900 multifocal intraocular lens: case series. J Cataract Refract Surg 2007; 33(12): 2067-2071.

4 Gills JP. Sutured piggyback toric intraocular lenses to correct high astigmatism. J Cataract Refract Surg 2003; 29(2): 402-404.

5 Hull CC, Liu CSC, Sciscio A. Image quality in polypseudophakia for extremely short eyes. $\mathrm{Br} J$ Ophthalmol 1999; 83: 656-663.
6 Kuryan J, Channa P. Refractive surgery after corneal transplant. Curr Opin Ophthalmol 2010; 21(4): 259-264.

7 Park JC, Mundasad MV, Tole DM. Piggyback intraocular lens implant to correct severe refractive error after penetrating keratoplasty in pseudophakes. Br J Ophthalmol 2009; 93(2): 272.

8 Shugar JK, Schwartz T. Interpseudophakos Elschnig pearls associated with late hyperopic shift: a complication of piggyback posterior chamber intraocular lens implantation. J Cataract Refract Surg 1999; 25: 863-867.

9 Spencer TS, Mamalis N, Lane SS. Interlenticular opacification of piggyback acrylic intraocular lenses. J Cataract Refract Surg 2002; 28(7): 1287-1290. 